ein Zwischenergebnis der bisherigen Debatte sein - muss als eine MehrebenenKonzeption formuliert werden. Das bedeutet: Eine solche Konzeption muss die direkte Arbeitsumwelt des Einzelnen, das gesamte Unternehmen sowie die Arenen der Wirtschaftspolitik als Räume einer Demokratisierung von Arbeit wahrnehmen; und sie muss die Einzelforderung für diese Räume zu einer Gesamtkonzeption kombinieren und über die Wechselwirkungen der Entwicklungen in den unterschiedlichen Ebenen nachdenken. Demokratische Ar- beit erfordert Demokratiepolitik am Arbeitsplatz, im Unternehmen und in der Gesellschaft.

Nicht zuletzt die Realisierungsvoraussetzungen demokratischer Arbeit machen deutlich, dass eine arbeitskraftzentriertinnovative Arbeitspolitik über die Arenen des Betriebs hinaus auf die Branchen-, Struktur- und Regionalpolitik erweitert werden muss. Der von der IG Metall vorgeschlagene Beteiligungsfonds zur Unternehmenssicherung könnte einen Weg der öffentlich finanzierten und kontrollierten In- vestitionssteuerung eröffnen, auf dem auch die arbeitspolitischen Entwicklungspfade beeinflusst werden können. ${ }^{6}$ Ohne eine Stärkung solcher wirtschaftsdemokratischen Einflussmöglichkeiten ist eine grundlegende Abkehr vom finanzmarktdominierten Typus der Unternehmens- und Wirtschaftspolitik schwer vorstellbar.

6 Vgl. IG Metall Vorstand (2009): Aktiv aus der Krise - Gemeinsam für ein Gutes Leben. Aktionsplan der IG Metall, Frankfurt, März, download: www.igmetall.de.

\title{
Schuldenfalle Hartz IV
}

\section{ÜBERSCHULDUNG - EIN VERMITTLUNGSHEMMNIS}

Wer überschuldet ist, tut sich meist sehr schwer damit, einen Arbeitsplatz zu finden. Dies liegt nicht nur an dem bei Überschuldeten häufig zu beobachtenden geringen Selbstbewusstsein, sondern auch daran, dass viele Arbeitgeber mit überschuldeten Mitarbeitern schlicht nichts zu tun haben wollen. Die erste Hürde, an der viele bereits scheitern, ist der bei Bewerbungen übliche Fragebogen. Hier findet sich oftmals die Frage, ob Lohnpfändungen zu erwarten seien. Auch in Vorstellungsgesprächen wird diese Frage gerne gestellt. Da die meisten Menschen viel ehrlicher sind als oft unterstellt wird, beantworten sie eine solche Frage meist wahrheitsgemäß - mit der Folge, dass eine Einstellung in der Regel nicht erfolgt.

Doch auch über denjenigen, die das Glück haben, ein Bewerbungsverfahren erfolgreich zu bestehen, ohne dass ihre Überschuldung thematisiert worden ist, hängt weiterhin ein Damoklesschwert: die Lohnpfändung, die sie den Arbeitsplatz kosten kann. Zwar ist eine Lohnpfändung - von Ausnahmen abgesehen - kein zulässiger Kündigungsgrund; da jedoch ein Arbeitgeber während der Probezeit ohne Angabe von Gründen kündigen kann und es keinerlei gesetzliche Verpflichtung gibt, befristete Arbeitsverträge zu verlängern, können Lohnpfändungen in der Praxis letztlich zum Verlust des Arbeitsplatzes führen. Grund dafür ist zum einen der Aufwand, der bei Lohnpfändungen auf den Arbeitgeber zukommt. Mit Eingang der Lohnpfändung wird er dem Gläubiger seines Arbeitnehmers gegenüber haftbar, den pfändbaren Teil des Lohnes an diesen abzuführen. Da die Pfändungsregeln der Zivilprozessordnung (ZPO) ein Musterbeispiel von vorsichtig ausgedrückt - gesetzlicher Komplexität sind, gerät jeder Arbeitgeber, der sich in dieser Materie nicht sehr genau auskennt, in die Gefahr, Fehler zu machen, für die er unter Umständen dem Gläubiger gegenüber geradestehen muss. Da die meisten Überschuldeten nicht nur einen, sondern mehrere Gläubiger haben, gibt es Arbeitnehmer mit einer ganzen Reihe von Lohnpfändungen, was insbesondere kleine Betriebe, denen es an Erfahrung mit dieser Materie fehlt, viel und für sie unproduktive Arbeitszeit kostet.

Das Stigma der Lohnpfändung kann weitere Schwierigkeiten mit sich bringen. Arbeitnehmerinnen und Arbeitnehmer, die mit viel Bargeld zu tun haben, wie zum Beispiel Kassiererinnen oder Bankangestellte, können unter Umständen, wenn eine Lohnpfändung eingeht, auch nach der Probezeit ihren Arbeitsplatz verlieren. Oder sie werden, falls tatsächlich einmal Geld fehlt, als erste verdächtigt, in die Kasse gegriffen zu haben. In kleineren Betrieben, in denen sich eine Lohnpfändung schnell herumspricht, werden überschul- dete Mitarbeiterinnen und Mitarbeiter auch oft gemobbt, da es als Makel gilt, sein Finanzgebaren nicht im Griff zu haben.

Dass Überschuldung ein Vermittlungshemmnis in Arbeit ist und sich durch eine entsprechende Beratung meist in den Griff bekommen lässt, hat inzwischen auch der Gesetzgeber erkannt. So wurde im Sozialgesetzbuch II (SGB II), besser bekannt als „Hartz IV“, die Schuldnerberatung als sogenannte Eingliederungsleistung definiert ( $\$ 16$, Abs. 2 SGB II). Auf den ersten Blick wirkt Hartz IV in diesem Punkt sehr vernünftig, gibt es doch viele positive Beispiele von ehemals langzeitarbeitslosen Menschen, die während oder nach ihrer Beratung durch eine Schuldnerberatungsstelle einen Arbeitsplatz gefunden haben. Auf den zweiten Blick erweist sich Hartz IV jedoch als moderne Version der aus der griechischen Mythologie bekannten Hydra, jenem Schlangenwesen, dem, wenn ihm ein Kopf abgeschlagen wurde, zwei Köpfe nachwuchsen. Prosaischer ausgedrückt: Hartz IV selbst ist inzwischen zur Schuldenfalle geworden.

Martin Staiger ist evangelischer Theologe und Sozialarbeiter, er arbeitet als Schuldnerberater, Referent und freier Journalist, u.a. für die Blätter für deutsche und internationale Politik (Berlin) und für Publik-Forum (Oberursel).

e-mail: majo.staiger@web.de 


\section{SCHULDENFALLE REGELSATZ}

Dass die Hartz-IV-Regelsätze nicht zum Leben reichen, ist bekannt. Dies thematisieren verschiedene Wohlfahrtsverbände wie der Deutsche Paritätische Wohlfahrtsverband oder die Diakonischen Werke seit Jahren, ohne dass sich etwas zum Positiven geändert hätte. Im Gegenteil: Der HartzIV-Regelsatz für einen Erwachsenen, der im Jahr 2005 bei $345 €$ pro Monat lag, stieg seitdem um ganze $6 €$. So leben Millionen von Hartz-IV-Empfängerinnen und -Empfängern weit unter der Armutsgrenze und damit in einer wirtschaftlichen Situation, die meist nur für begrenzte Zeit haltbar ist. Anfangs helfen vielleicht noch kleine Ersparnisse oder Besitzstände wie Kleidung und Mobiliar, die Situation zu überbrücken. Sind die Reserven aufgezehrt, wird es finanziell äußerst eng. Lassen sich Ersatzanschaffungen nicht mehr länger hinausschieben, wird vielleicht das Konto überzogen, falls die Bank nicht den Dispositionskredit schon gesperrt hat. Oder man kann eine Telefonrechnung, das Zeitungsabonnement, die Prämie der Haftpflichtversicherung und ähnliches nicht mehr bezahlen.

Besonders dramatisch sind oft die Folgen der Jahresabrechnungen der Energieversorgungsunternehmen. So kann schon eine Stromnachforderung von $100 €$ oder $150 €$ zum Einstieg in die Schuldenspirale führen. Da die Energieversorgungsunternehmen bei Zahlungsverzug recht schnell mit der Abschaltung des Stroms drohen, setzen die meisten Menschen alles daran, Stromschulden zügig zu begleichen. Das Geld fehlt dann an anderer Stelle. Wenn der Kunde die Nachforderung nicht bezahlen kann und die Stromabschaltung in Aussicht steht, sind die für Hartz-IV-Empfänger zuständigen, sogenannten Jobcenter auf Antrag hin verpflichtet, die Rechnung zu begleichen - zumindest als Darlehen. Zur Tilgung behalten die Jobcenter bis zu $10 \%$ der Regelleistung ein. So wird das ohnehin zu knappe Geld noch einmal knapper. Vielleicht borgt man sich dann etwas bei Nachbarn oder Verwandten, um das eine oder andere Loch zu stopfen, oder man lässt im Lebensmittelladen anschreiben. Die Schuldenspirale beginnt sich zu drehen: Mahnungen, Drohungen, Inkassoschreiben, Vollstreckungsbescheide, Gerichtsvollzieherbesuche und Pfändungen. Der Schuldenberg wächst rasch - und vielfach auch die Verzweiflung und die Anfälligkeit für schwere Krankheiten. All das sind denkbar schlechte Voraussetzungen, um erfolgreich nach Arbeit zu suchen.

\section{SCHULDENFALLE WOHNKOSTEN}

„Leistungen für Unterkunft und Heizung werden in Höhe der tatsächlichen Aufwendungen erbracht, soweit diese angemessen sind“. So lautet $\$ 22$, Satz 1 SGB II. Dieser Satz mag harmlos klingen. Er hat es aber in sich. Denn viele Kommunen bzw. Landkreise haben Angemessenheitsgrenzen festgelegt, die fern jeder Realität sind. So gibt es eine ganze Reihe von Wohngebieten, in denen die tatsächlichen Wohnkosten von den Jobcentern nicht einmal annähernd anerkannt werden. Wer nicht nur übergangsweise von Hartz IV leben muss, gerät so, falls er nicht sehr schnell eine andere Wohnung findet, bald in Mietrückstände und muss im schlimmsten Falle irgendwann mit einer Räumungsklage rechnen.

Nach der juristischen Theorie unterliegt „die Angemessenheit der zu berücksichtigenden Unterkunftskosten ... als unbestimmter Rechtsbegriff in vollem Umfang der gerichtlichen Kontrolle". ${ }^{1}$ In der Praxis sieht dies jedoch oft anders aus. Denn die meisten Arbeitslosengeld-IIEmpfänger scheuen sich davor, mit der Behörde, von der sie existenziell abhängig sind, einen Rechtsstreit zu führen. Wer es dennoch wagt, hat vor den örtlichen Sozialgerichten oft keinen Erfolg, da es die meist mehr als ausgelasteten Sozialrichter zeitlich schlicht überfordert, sich mit der Wohnkostensituation einer Kommune oder eines Landkreises intensiv zu beschäftigen. So behilft sich mancher Richter damit, ein bisschen im Internet zu recherchieren, ob er in der Umgebung des Klagenden die ein oder andere ,angemessene“ Wohnung findet, und wenn dies der Fall ist, die Klage abzuweisen. Da die wenigsten vor den örtlichen Sozialgerichten Gescheiterten vor das Landessozialgericht oder gar vor das Bundessozialgericht ziehen, ist in der Praxis die „Angemessenheit“ der zu berücksichtigenden Unterkunftskosten nur in sehr eingeschränktem Umfang der gerichtlichen Kontrolle unterworfen.

Hartz-IV-Empfänger, die in einer ,unangemessenen" Wohnung leben, bekommen in der Regel zu Beginn des Leistungsbezuges für eine Übergangszeit die Miete vollständig bezahlt. Relativ bald erhalten sie dann jedoch ein Schreiben des Jobcenters mit der Aufforderung, sich um eine kostengünstigere Wohnung zu bemühen oder andere Möglichkeiten zur Senkung der Kosten zu suchen. Wer in einer ,unangemessenen “Wohnung lebt und keine, ,angemessene " findet, was in Ballungsräumen eher die Regel als die Ausnahme ist, muss damit rechnen, über kurz oder lang Mietschulden anzuhäufen oder gar seine Bleibe zu verlieren, falls er der Behörde nicht permanent und akribisch seine erfolglose Wohnungssuche nachweist, was nur den wenigsten Menschen wirklich gelingt. ${ }^{2} \mathrm{Da}$ in Deutschland alles Mögliche statistisch erfasst wird, nicht aber die Zahl der Wohnungsverluste aufgrund von Mietschulden, kann man über das Ausmaß der im $\mathrm{Zu}$ sammenhang mit Hartz IV entstehenden Wohnungslosigkeit nur spekulieren. Es gibt jedoch zahlreiche Anhaltspunkte dafür, dass die Zahl der Wohnungsverluste seit Hartz IV deutlich angestiegen ist.

Auch wer eine Wohnung innerhalb der örtlichen „Angemessenheitsgrenzen“ findet, hat gute Chancen, sich dennoch verschulden zu müssen. Denn die bei einem Neueinzug meist fällige Mietkaution oder die Geschäftsanteile für die Mitgliedschaft in einer Baugenossenschaft kann ein Hartz-IV-Empfänger selten bezahlen. Hier hilft nach den Bestimmungen des Sozialgesetzbuches II das Jobcenter aus - jedoch nur auf Darlehensbasis. Weitere Schulden entstehen unter Umständen beim alten Vermieter, da billige Wohnungen oft so kurzfristig angeboten werden, dass aufgrund der dreimonatigen Kündigungsfrist vorübergehend doppelte Miete bezahlt werden muss, die auch nicht in jedem Fall von den Jobcentern übernommen wird.

\section{SCHULDENFALLE BEHÖRDENHANDELN}

Die Zentrale der Bundesagentur für Arbeit erstellt einmal jährlich einen sogenannten Planungsbrief, der den Jobcentern Vorgaben macht, wie sie im nächsten Jahr zu arbeiten haben. Eines der wichtigsten Ziele des Planungsbriefes für 2009 ist eine Einsparung bei den „passiven Leistungen“ " um 6,7\%. Mit den „passiven Leistungen“ man beachte den Begriff, mit dem das Stammtischgerede von der „Sozialen Hän-

\footnotetext{
Münder, J. (Hrsg.) (2005): Sozialgesetzbuch II. Grundsicherung für Arbeitsuchende. Lehr- und Praxiskommentar, Baden-Baden, Randziffer 23 zu $\S 22$ SGB II.

2 Vgl. hierzu ausführlich Staiger, M. (2006): HartzIV-Unterkunft, in: Blätter für deutsche und internationale Politik 5, S. 524-527.
} 
gematte" im offiziellen Sprachgebrauch der Bundesagentur für Arbeit angekommen ist - sind die Leistungen zum Lebensunterhalt für Erwachsene und für Kinder nach $\$ \$ 20 f f$. SGB II gemeint. Diese Ausgaben lassen sich nur dann um 6,7 \% senken, wenn man über einige Bestimmungen des Sozialgesetzbuches, wie z. B. die Pflicht zu Aufklärung und Beratung ( $\$ 13$ und $\$ 14$ SGB I) oder die Pflicht ,alle für den Einzelfall bedeutsamen, auch die für die Beteiligten günstigen Umstände zu berücksichtigen“ ( $\$ 20$ SGB X), großzügig hinweggeht. So wird die Umsetzung der Nürnberger Planungsprozesse zu einem weiteren Risiko für Hartz-IV-Empfänger, in die Schuldenfalle zu geraten.

Die Informationen, die aus den Jobcentern nach außen dringen, zeugen von einer großen Phantasie, mit welchen Mitteln die Nürnberger Einsparvorgaben vor Ort umgesetzt werden. Da werden z. B. Mitarbeiterinnen und Mitarbeiter unter Druck gesetzt, eine Mindestquote von Sanktionen zu verhängen, die Leistungsabsenkungen von mindestens $30 \%$ vorsehen, oder sie werden angewiesen, für „mehrtägige Klassenfahrten im Rahmen der schulrechtlichen Bestimmungen", die nach $\$ 23$, Abs. 3, Satz 3 SGB II in voller Höhe bezahlt werden müssen, nur einen Teil der Kosten zu gewähren. Oder es werden im Gegensatz zu der in diesem Punkt völlig eindeutigen Rechtsprechung, dass Heizkosten nur dann nicht in voller Höhe $\mathrm{zu}$ bezahlen sind, wenn ein eindeutig verschwenderisches Heizverhalten vorliegt, lediglich Heizkostenpauschalen gewährt, sodass Bewohnerinnen und Bewohner schlecht isolierter Wohnungen auf einem Teil der Heizkosten sitzen bleiben. Sehr beliebt ist anscheinend auch die Vermittlung in - vorsichtig ausgedrückt - nicht unbedingt passgenaue Weiterbildungsmaßnahmen, was für die Jobcenter einen doppelten Charme hat: Denjenigen, die das Angebot nicht annehmen, können die Bezüge gekürzt werden. Und wer die Schulung mitmacht, fällt solange aus der Arbeitslosenstatistik heraus.

Auch die wochen- oder gar monatelangen Bearbeitungszeiten, die eine Folge der komplizierten Gesetzgebung, der permanenten personellen Unterbesetzung bei den meisten Jobcentern, der hohen Fluktuation sowie der mangelnden Ausbildung vieler Mitarbeiterinnen und Mitarbeiter sind, führen viele Menschen in die Schuldenfalle. Mehr als vier Jahre nach Verab- schiedung von Hartz IV werden noch immer sehr viele rechtswidrige Leistungsbescheide versendet, erkennbar mittellose Menschen müssen oft viele Wochen auf die erste Arbeitslosengeld-II-Zahlung warten und es werden erbrachte Leistungen häufig ohne Prüfung, ob das gesetzlich überhaupt zulässig ist, - zurückgefordert. Vielfach haben Langzeitarbeitslose auch schlicht Angst vor der Behörde, die ihnen doch eigentlich helfen sollte. Sie reagieren mit Rückzug, was zu Meldeversäumnissen oder Verstößen gegen die Pflicht, sich kontinuierlich zu bewerben, führen kann. Das schafft den Jobcentern Anlässe, die ohnehin zu einem menschenwürdigen Leben nicht ausreichenden SGB-II-Leistungen zu kürzen. Für die Betroffenen bedeuten solche Sanktionen existenzielle Nöte und die Gefahr, in die Schuldenfalle zu geraten.

\section{SCHULDENFALLE ARBEIT}

Die meisten Menschen, die aus dem HartzIV-Bezug heraus Arbeit finden, haben bereits einen Monat nach dem Neustart ins Berufsleben Schulden beim Jobcenter. Dies liegt daran, dass sie die im Monat der Arbeitsaufnahme erhaltenen Hartz-IV-Leistungen zurückzahlen müssen, wenn sie ihren ersten Arbeitslohn, der in der Regel am Monatsende gezahlt wird, erhalten. Der rechtspolitische Unsinn, ex-langzeitarbeitslose Menschen mit Schulden ins Berufsleben zu entlassen, ist rechtlich korrekt. Nach der vom Bundesverwaltungsgericht im Jahr 2004 entwickelten „Zuflusstheorie“, ist „,Bedarfszeitraum', in Bezug auf den die Hilfe zu berechnen und innerhalb dessen zufließendes Einkommen als Einkommen zu berücksichtigen ist, grundsätzlich der jeweilige Kalendermonat. ... Auch Einkommen, das regelmäßig erst zum Ende des Kalendermonats zufließt, ist grundsätzlich nur als Einkommen des Kalendermonats anzurechnen, in dem es tatsächlich zugeflossen ist". 3

Der Gesetzgeber hätte es in der Hand, solche Fallstricke zu beseitigen. Er müsste nur die erste Entwurfsfassung zum SGB II in Kraft setzen. In dieser war vorgesehen, Einnahmen der letzten fünf Kalendertage eines Monat dem Folgemonat zuzurechnen. ${ }^{4}$ So ließe sich vermeiden, dass Ex-Arbeitslose, die Arbeit gefunden haben, mit Schulden in ihre neue Arbeit starten. Aber wie so oft in der Sozialgesetzgebung der letzten Jahre haben sich auch bei diesem Detail die weniger Weitblickenden durch- gesetzt, die noch nie etwas davon gehört haben, dass der vordergründig etwas teurere Weg langfristig oftmals der wesentlich billigere ist. Denn ein schuldenfreier Mensch arbeitet in der Regel wesentlich motivierter als ein verschuldeter. Außerdem wird er weniger krank und verliert seltener seine Arbeitsstelle.

\section{FAZIT}

Gäbe es im Deutschen ein Wort für das Gegenteil von Nachhaltigkeit, ließe sich Hartz IV auf den Begriff bringen. In der Geschichte des Sozialgesetzbuches gab es selten eine so kurzsichtige Reform. Und selten wurde ein Gesetz vom deutschen Bundestag verabschiedet, dem es so offenkundig an Wissen über die Arbeitswelt wie auch an Menschenkenntnis fehlt. Es kann doch wirklich niemand ernsthaft glauben, dass eine nennenswerte Zahl von unter der Armutsgrenze lebenden und von überforderten Behörden gegängelten Menschen so stabil ist, auf dem hart umkämpften Arbeitsmarkt erfolgreich eine existenzsichernde Arbeit zu finden. Es kann auch niemand ernsthaft glauben, dass die meisten derjenigen Ex-Hartz-IV-Empfänger, die trotz dieser Widrigkeiten eine Arbeitsstelle gefunden haben, sie auch auf Dauer behalten werden, wenn die Jobcenter womöglich über eine Lohnpfändung Hunderte oder gar Tausende von Euro von ihnen zurückfordern. Solange aber nicht nur am Stammtisch, sondern auch in der Bundespolitik die Mentalität vorherrscht, Langzeitarbeitslosigkeit sei vor allem ein Ergebnis individuellen Versagens, das es entsprechend zu bestrafen gilt, ist $\mathrm{zu}$ befürchten, dass sich an den kleingeistigen Bestimmungen des Sozialgesetzbuches II nichts ändern wird.

Die Hoffnung stirbt jedoch bekanntlich zuletzt. Vielleicht birgt der gegenwärtige Wirtschaftsabschwung ja zumindest die Chance, zu erkennen, dass Arbeitslosigkeit in erster Linie konjunkturbedingt und nicht individuell verschuldet ist. Eine solche Einsicht könnte vielleicht zu einer realitätsnahen SGB-II-Reform führen, die das Abrutschen von Langzeitarbeitslosen in die Schuldenfalle verhindert.

\footnotetext{
3 BverwG, Urteil vom 22.04.2004, Aktenzeichen 5 C 68.03, www.bverwg.de/media/archive/2030.pdf 4 Vgl. Münder, J. (Hrsg.) (2005): Sozialgesetzbuch II. Grundsicherung für Arbeitsuchende. Lehr- und Praxiskommentar, Baden-Baden, Randziffer 53 zu §11 SGB II.
} 